\section{RESEARCHES ON THE RADYOMETER}

\section{By Prof. Paul Volpicelli.}

I. AL radiometers do not possess the same sensibility A necessary for every experiment.

2. The most sensitive of the two which are in the physical museum of the Roman University shows that the freezing mixture of chloride of sodium and snow, applied to the upper hemisphere of the small globe, produces a rotation of the mill in the same direction in which it is produced by heat radiation, $i . \varepsilon$., with the white face of the small discs in advance.

3. If to this lowering of temperature be added a radiation of heat, the rotation of the apparatus is accelerated at the same time.

4. If the freezing mixture referred to be placed on the lower hemisphere of the same small globe, the apparatus will rotate with the absorbing, i.e., the black faces in advance, and consequently in the direction contrary to that of the preceding experiment, i.e., to the direction produced, if to the same lower hemisphere, radiant heat be applied.

5. If during the rotation produced by the application of the freezing mixture to the lower hemisphere of the small globe we cause radiant heat to strike the same globe, the apparatus will be brought to a stop; and as soon as the source of heat is withdrawn, the rotation will immediately commence.

6 . If the small globe is plunged entirely in a heated liquid, or even in a freezing mixture, the apparatus will remain at rest.

7. It should be noted that the freezing mixture applied to the upper hemisphere of the small globe, produces a rotation in the direction opposite to that produced by the same mixture when applied to the lower hemisphere.

8 It has been stated that the radiometer in darkness remains at rest ; but it should be remarked that if even in darkness it is affected by dark radiant heat, the apparatus will assume a rotatory movement; yet the instrument may remain at rest even when placed in a dark space.

9. The luminous rays of the full moon focussed by means of a lens, do not cause rotation of the instrument.

10. If the radiation of the flame of a Locatelli lamp is caused to traverse several plates of perfectly transparent glass, it will be seen by the number of turns of the instrument, that the law of De la Roche is verified regarding the absorption of radiant heat through these plates, however many they may be. I have been able by this means to diminish the radiant heat to such an extent as to cause the rotation of the radiometer to cease, although the light of the same radiation was increased by means of a lens.

ix. The same radiation, that, viz., produced by Locatelli's lamp, by traversing a saturated but perfectly transparent solution of alum, before reaching the radiometer, did not set it in motion, although the radiant light was but little diminished; and the same is the case when the light is increased.

12. It would appear at present that the rotation of the radiometer depends on radiant heat and not on the lumincus rays.

13. It appears also that the mechanical cause of the rotation of the radiometer consists in the motion of the molecules of very rarefied gas in the small globe, which is in accordance with the opinion of modern thermodynamics.
THE SIPHON RECORDER AND AUTOMATIC CURB SENDER

FOR some time after the introduction of submarine telegraphy Sir William Thomson's mirror galvanometer was the only instrument delicate enough to receive the signals transmitted through a long cable. The spot of light reflected from the mirror moves over the scale and indicates every change of current in the cable. The clerks by degrees learn to interpret the motions of the spot of light, and are able to read the signals sent. The signals, however, must be read at the instant of arrival, and the clerk has no way of correcting what he receives

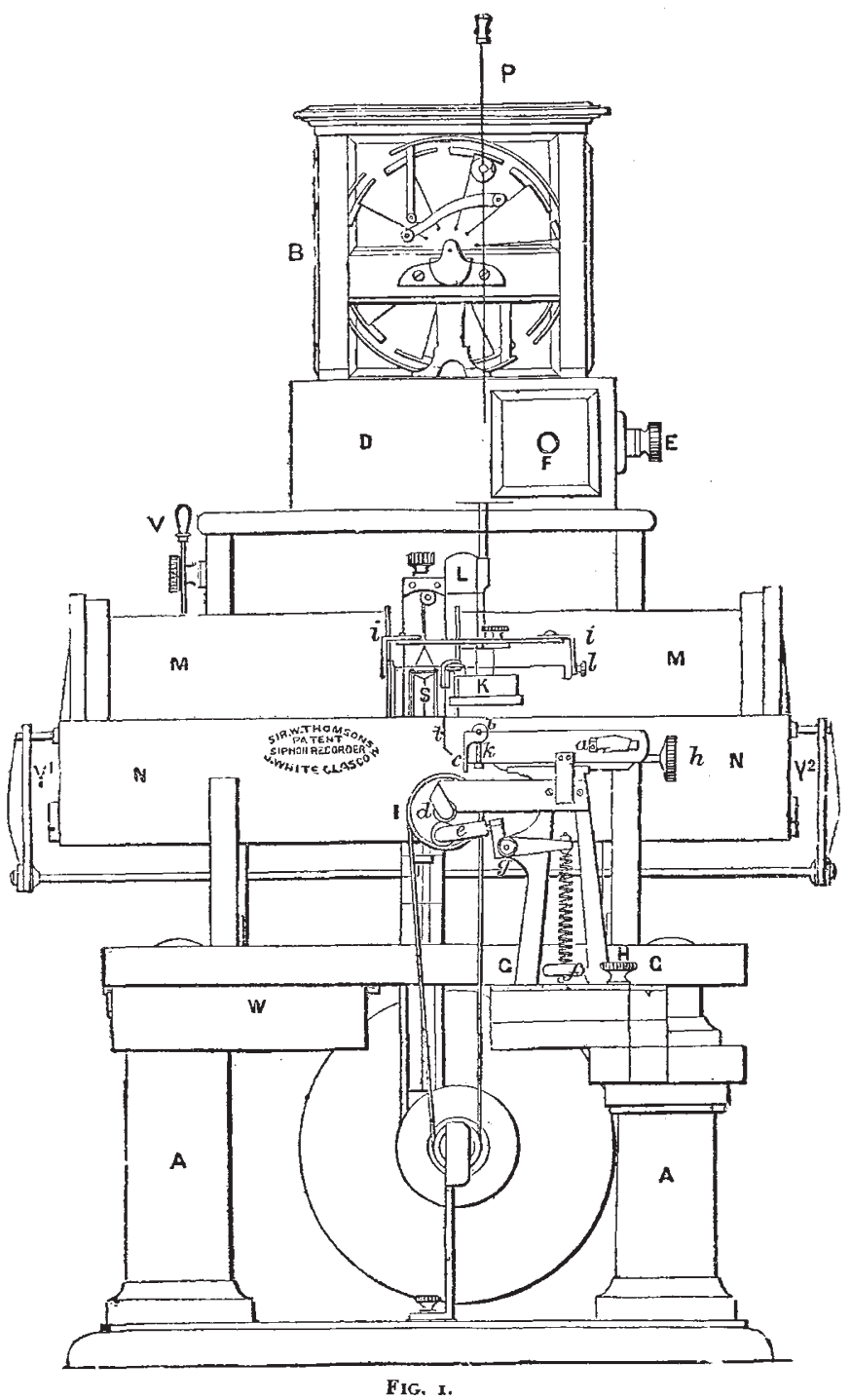

except by having the signals repeated from the distant end.

The Siphon Recorder was devised, more recently by Sir William Thomson, for the purpose of receiving and recording the signals transmitted through a submarine cable; though it may also be used for receiving signals sent along a land line. It actually draws on paper the curves corresponding to the changes of current which pass through the line. Thus a permanent record is made of every signal that is sent, and not only can the clerk be sure that he reads the signals correctly, but also any 